\title{
Effect of beraprost sodium on arterial stiffness in patients with type 2 diabetic nephropathy
}

\author{
Ki Young $\mathrm{Na}^{1,2}$, Dong Ki Kim²,3, Sung Gyun Kim ${ }^{4}$ Young-Ki Lee ${ }^{5}$ and Chun Soo Lim²,6*
}

\begin{abstract}
Background: Diabetic nephropathy is the leading cause of end-stage renal disease (ESRD). Cardiovascular (CV) complications are the most common cause of death among ESRD patients. Beraprost sodium (BPS) is a prostacyclin analog with vasodilatory and antiplatelet effects.

Methods: This is a multicenter prospective, randomized, double-blind, placebo-controlled trial to determine whether treatment with BPS improves arterial stiffness in patients with type 2 diabetic nephropathy. A total of 102 participants with type 2 diabetic nephropathy will be screened, enrolled, and randomly assigned to receive either $80 \mu \mathrm{g}$ BPS or placebo daily for 12 weeks. The primary outcome is the change in brachial-ankle pulse wave velocity between baseline and after 12 weeks of medication use. The secondary outcomes will include changes in the ankle-brachial index, the urine albumin to creatinine ratio, the estimated glomerular filtration rate, lipid profiles, and blood pressure from baseline to after treatment.
\end{abstract}

Discussion: This clinical trial is the first to investigate the effects of BPS on changes in CV biomarkers, albuminuria, renal function, and lipid profiles in patients with diabetic nephropathy.

Trial registration: ClinicalTrials.gov number NCT01796418

Keywords: Beraprost sodium, Arterial stiffness, Diabetic nephropathy, Cardiovascular, Pulse wave velocity

\section{Background}

The impact of diabetic nephropathy on the increasing population with chronic kidney disease (CKD) and end-stage renal disease (ESRD) is enormous. Diabetic nephropathy has become the leading cause of ESRD and is responsible for more than $40 \%$ of all cases in the USA and South Korea [1,2]. Although randomized studies have demonstrated that intensified multifactorial intervention in patients with type 2 diabetes reduces the risk of microangiopathy, cardiovascular $(\mathrm{CV})$ events, and mortality [3-5], the incidence of ESRD due to diabetes is relentlessly increasing worldwide. CV complications are the number one cause of death among patients with ESRD [1,2]. Moreover, CV risk is not limited to ESRD, and the risk of $\mathrm{CV}$ mortality begins to increase with even a mild impairment of kidney function [6]. Therefore, early

\footnotetext{
*Correspondence: cslimjy@snu.ac.kr

${ }^{2}$ Department Internal Medicine, Seoul National University College of Medicine, 103 Daehak-ro, Jongno-gu, Seoul 110-799, South Korea

${ }^{6}$ Seoul National University Boramae Medical Center, 20 Boramae-ro 5-gil,

Dongjak-gu, Seoul 156-707, South Korea

Full list of author information is available at the end of the article
}

detection and intervention for $\mathrm{CV}$ complications are important to reduce mortality among patients with CKD or ESRD due to diabetes. In this regard, surrogate markers precisely estimating CV risk are essential. Pulse wave velocity (PWV) and the ankle-brachial index (ABI) are useful noninvasive biomarkers for predicting $\mathrm{CV}$ risk [7]. PWV provides a measure of large artery stiffness and has been reported as a risk factor for nonfatal $\mathrm{CV}$ disease and $\mathrm{CV}$ mortality independent of systolic blood pressure in dialysis patients $[8,9]$. The ABI is a clinical tool for the detection of peripheral arterial disease and the prediction of CV mortality and morbidity [10].

Beraprost sodium (BPS) is a stable orally active prostacyclin $\left(\mathrm{PGI}_{2}\right)$ analog. BPS acts by binding to $\mathrm{PGI}_{2}$ membrane receptors and inhibiting the release of $\mathrm{Ca}^{2+}$ from intracellular storage sites. These effects lead to the relaxation of the smooth muscle cells and vasodilation. BPS has also been suggested to improve microvascular circulation through the reduction of red blood cell deformability. Moreover, BPS alleviates vascular thrombosis by inhibiting platelet aggregation. Through 
these vasodilatory and antiplatelet effects, BPS appears to be an effective agent in the treatment of patients with pulmonary arterial hypertension, Buerger's disease, and arteriosclerosis obliterans [11].

An in vitro experiment showed that BPS improved endothelial function through the induction of nitric oxide (NO) production and endothelial NO synthase activation in endothelial cells [12]. In addition, BPS lowered serum vascular cell adhesion molecule-1 levels and prevented the increase of carotid intima-media thickness in patients with type 2 diabetes, which suggested a beneficial effect of BPS on the progression of atherosclerosis in diabetic patients [13]. The development of arterial stiffness, as assessed by PWV, has been prevented by BPS treatment in elderly patients with cerebral infarction $[14,15]$. BPS has also been shown to improve dyslipidemia and metabolic syndrome in diabetic animal models $[16,17]$. A recent study has reported that 8 weeks BPS treatment improves pain in diabetic patients with painful peripheral neuropathy [18]. However, no studies have observed the effects of BPS on $\mathrm{CV}$ biomarkers in diabetic nephropathy patients with a high risk of CKD or ESRD.

Based on reports of the beneficial effects of BPS, we hypothesize that BPS treatment will improve arterial stiffness in diabetic nephropathy patients. To evaluate this hypothesis, we will conduct this clinical trial in patients with type 2 diabetic nephropathy.

\section{Methods}

Hypothesis

Treatment with BPS will improve arterial stiffness in patients with type 2 diabetic nephropathy. Compared with placebo-treated patients, arterial PWV will decrease in BPS-treated patients.

\section{Study design}

This is a multicenter prospective, randomized, doubleblind, placebo-controlled trial. This study is an investigatorinitiated clinical trial. The study algorithm is shown in Figure 1. After enrollment and treatment, clinical follow-up will be conducted after 4 and 12 weeks of treatment.

\section{Study participants}

All patients aged 20 to 75 years with type 2 diabetes mellitus will be screened. The following will be performed at the initial visit: 1) questionnaire regarding past medical history and drug history; 2) physical examination of all systems; 3) measurement of height and weight; 4) blood pressure and pulse rate measurement; and 5) collection of blood and urine. Patients who meet all of the inclusion and exclusion criteria and provide written informed consent are eligible for this study (Table 1).

Serum and urine creatinine $(\mathrm{Cr})$ will be measured using the isotope dilution mass spectrometry-traceable method on a Toshiba TBA 200FR Analyzer (Toshiba, Tokyo, Japan). Urinary albumin will be measured using an immunoturbidimetric assay on a Toshiba TBA 120FR Analyzer. The estimated glomerular filtration rate (eGFR) is calculated using the Modification of Diet in Renal Disease formula. The formula was defined as follows, where the serum $\mathrm{Cr}$ concentration is in $\mathrm{mg} / \mathrm{dL}$ : $\mathrm{eGFR}=175 \times$ $\mathrm{Cr}^{-1.154} \times \mathrm{Age}^{-0.203} \times(0.742$ if female $)[19]$.

\section{Randomization}

A research coordinator will perform the randomization and deliver the study drugs. The participants and investigators will be blinded to the treatment assignment. A list of random numbers will be generated by an independent statistician and then used to randomly allocate the treatments to equally sized treatment and control groups. The randomization will be stratified on the basis of sex and age, which will utilize a randomized block design in blocks of four patients in the order of enrollment in each arm. A randomization code list will be generated by a third party - the medical statistics support team in the Seoul National University Boramae Medical Center. The treatments will be allocated to the participants in accordance with the predefined randomization list (1:1 ratio for the treatment and control groups).

\section{Treatments}

After randomization, the participants will take either a BPS (treatment group) or placebo (control group) pill during the study. The tablet shapes and packaging of the placebo pills are indistinguishable from those of the original BPS pills. The BPS and placebo tablets are provided by Astellas Pharma Korea, Inc. (Seoul, Korea). The prescription and administration of the study drugs will be performed in a double-blind manner. Based on the report showing the prevention of arterial stiffness with BPS [14], $80 \mu \mathrm{g} /$ day BPS in two divided doses will be administered to the participants for 12 weeks. The administration of other prostacyclin agents, such as limaprost alfadex, or fluorinated pyrimidine antifungal agents, such as flucytosine, will be prohibited during the study. Participants with any changes in antihypertensive, antiplatelet, or anticoagulant medications during the study will be dropped. Investigators or research coordinators will evaluate drug compliance in the study participants, and participants with less than $80 \%$ compliance will be dropped from the study.

\section{Outcome measures}

The primary outcome to be measured is the change in the brachial-ankle PWV (ba-PWV) between baseline and after 12 weeks of treatment. The secondary outcomes will include changes in the ABI, urine albumin to creatinine ratio, eGFR, lipid profiles, and blood pressure from baseline to after treatment. 


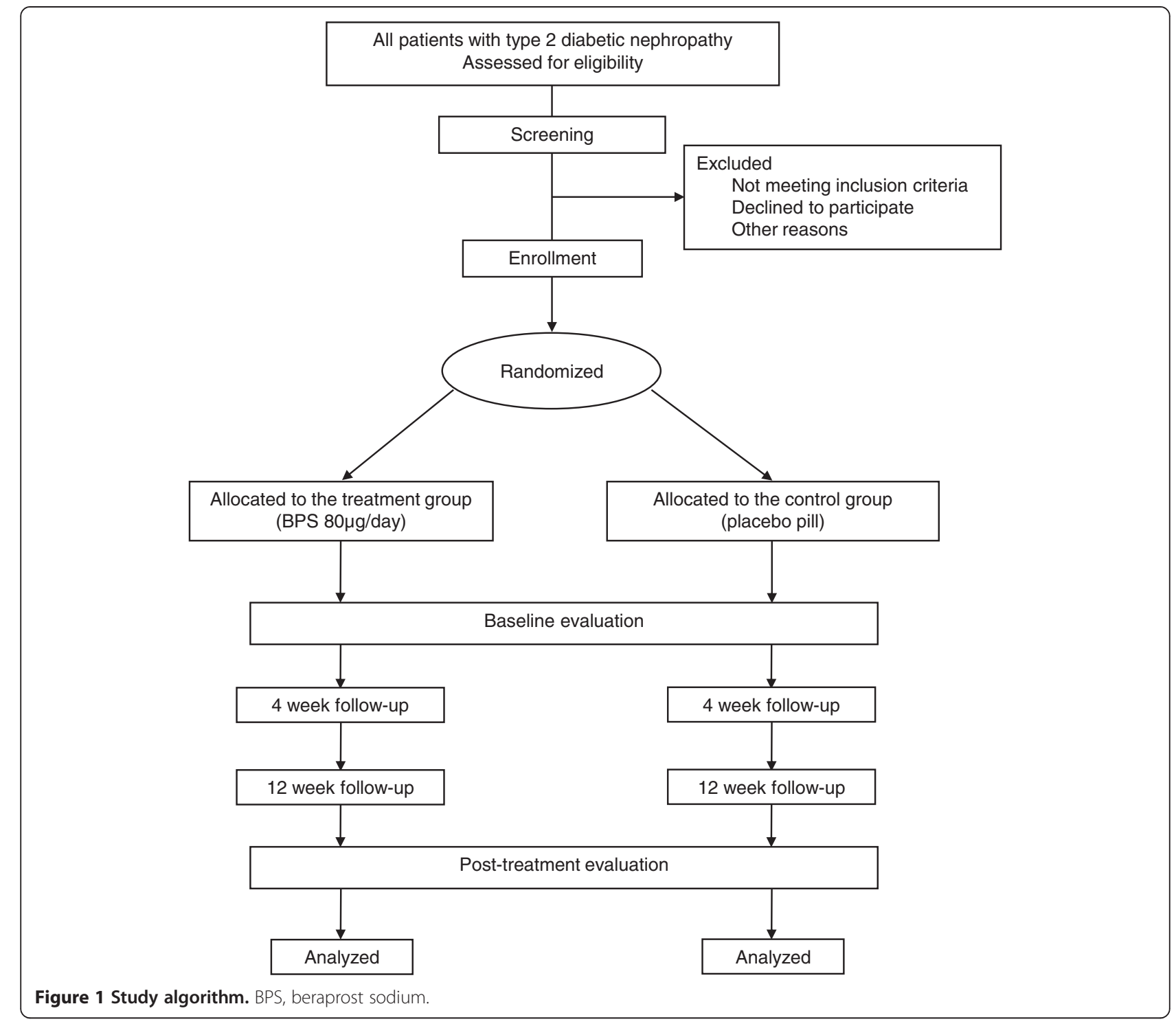

\section{Clinical and laboratory evaluations}

The physical examination and medication reviews will be performed before treatment and after 4 and 12 weeks of treatment. The laboratory evaluations, including a complete blood count, $\mathrm{Cr}$, total cholesterol, triglyceride, low-density lipoprotein cholesterol, random urine albumin, and urine $\mathrm{Cr}$, will be performed at baseline and after 12 weeks of treatment. ba-PWV and ABI will be measured using a Colin pulse waveform analyzer (Colin CO, Ltd, Komaki, Japan) at baseline and after 12 weeks of treatment. The study schedule is shown in Figure 2.

\section{Monitoring and safety assessments}

Before the start of this trial, a central monitor will review the study protocol and clinical research form (CRF) with the investigators and research coordinators. During the study period, a monitor will regularly visit each institute and evaluate the records of the participants, the CRF, compliance with the study protocol and Good Clinical Practice guidelines, and enrollment status. The storage, delivery, and accountability of the study drug will also be monitored during this period.

Any presence of bleeding, headache, hot flushes, shock, interstitial pneumonia, abnormal liver function, hypersensitivity reaction, angina, or gastrointestinal discomfort will be recorded during treatment with BPS/placebo. All adverse events, including serious adverse events (SAEs), will be recorded and followed-up for the duration of the study or until resolution. The assessment of adverse events will be performed by investigators and research coordinators. All SAEs will be graded and reported to the principal investigator and the ethics committee. 
Table 1 Inclusion and exclusion criteria

Inclusion criteria

Age between 20 and 75 years

Type 2 diabetes using a glucose-lowering agent or insulin

Estimated GFR by the IDMS MDRD equation $\geq 30 \mathrm{ml} / \mathrm{min} / 1.73 \mathrm{~m}^{2}$

More than two UACR $\geq 30 \mathrm{mg} / \mathrm{g}$ or UPCR $\geq 300 \mathrm{mg} / \mathrm{g}$ in the most recent 6 months

Blood pressure $\leq 140 / 90 \mathrm{mmHg}$ without any additional antihypertensive

medications in the most recent 3 months

\author{
Exclusion criteria \\ History of kidney transplantation \\ Advanced congestive heart failure ( $\geq$ NYHA class III) \\ Uncontrolled arrhythmia \\ Advanced liver cirrhosis (Child-Pugh class C) \\ History of bleeding diathesis \\ Active infection or uncontrolled inflammatory disorders \\ History of cerebrovascular accident or myocardial infarction \\ Current use of an anticoagulant \\ Current use of more than two antiplatelet agents \\ Advanced malignancy (life expectancy $\leq 6$ months) \\ Uncontrolled diabetes ( $\mathrm{HbA} 1 \mathrm{C}>10 \%)$ \\ Severe anemia (hemoglobin $<8.0 \mathrm{~g} / \mathrm{dL}$ ) \\ Pregnancy or lactation \\ Genetic disease including galactose intolerance, lactose \\ deficiency, and glucose-galactose malabsorption
}

GFR, glomerular filtration rate; HbA1C, hemoglobin A1C; IDMS, isotope dilution mass spectrometry; MDRD, Modification of Diet in Renal Disease; NYHA, New York Heart Association; UACR, urine albumin to creatinine ratio; UPCR, urine protein to creatinine ratio.

\section{Sample size calculations}

No previous report has evaluated the effect of BPS on arterial stiffness in diabetic patients. We therefore referred to a study showing that the mean and standard deviation of the PWV change due to BPS treatment in patients with a history of cerebrovascular accidents were $260 \mathrm{~cm} / \mathrm{s}$ and
$345 \mathrm{~cm} / \mathrm{s}$, respectively [14]. We adjusted the sample size for an estimated drop-out rate of $25 \%$ due to poor compliance, a two-sided level of significance of $\alpha=5 \%$, and a power of $1-\beta=90 \%$, which indicated that 51 participants will be required in each group to detect a significant difference with a two-sided Student's $t$ test [20]. A total

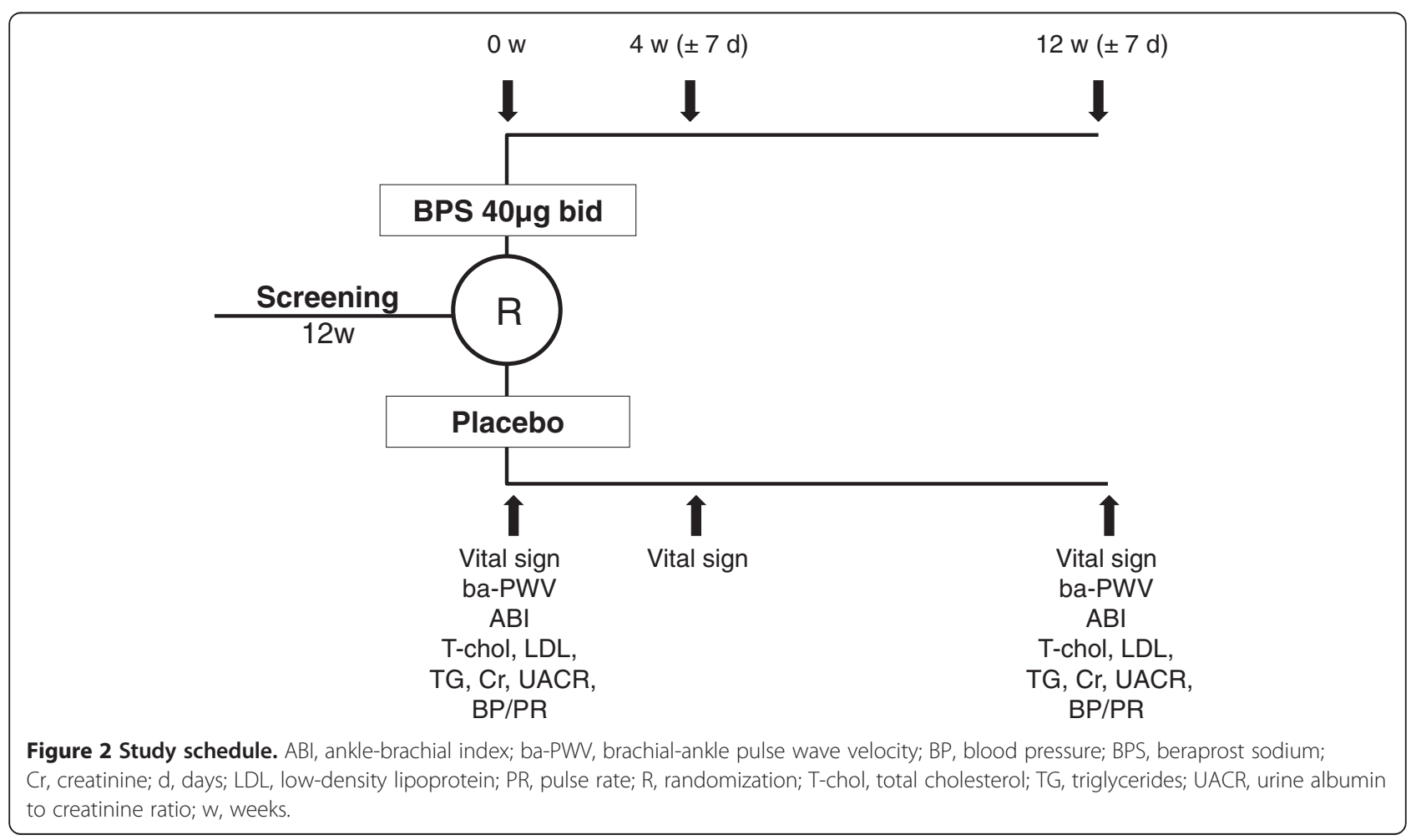


of 102 participants will be randomized and included in the analysis.

\section{Statistical analyses}

The statistical analyses will be performed both on per protocol (PP) and intention-to-treat (ITT) bases. For the PP analysis, all participants who completed the study and were evaluated for the primary outcome will be included. For the ITT analysis, all participants who signed the written informed consent and were randomized into the study will be included, regardless of whether the correct treatment was administered. To evaluate the primary outcome, a PP analysis will be performed for those participants who showed a primary outcome variable among the targets for the PP analysis. The secondary outcomes will be evaluated in the participants who completed the study. For the safety evaluation, all participants who signed the written informed consent and were randomized to receive at least one of the active treatments (BPS/placebo medication) will be included in the analysis. The safety analysis will include the calculation of the frequencies and rates of complications and SAEs reported in the two groups.

The baseline clinical characteristics and laboratory data of the study participants will be summarized in terms of frequencies and percentages for categorical variables and means with standard deviations for continuous variables. Continuous variables will be analyzed using Student's $t$ test or the Mann-Whitney U test. Chi-squared test or Fisher's exact test will be used for categorical variables. Differences in the changes in the outcome measurement variables between the treatment and control groups will be tested with one-way analysis of variance. Differences in the temporal changes in the outcome measurement variables in each group will be analyzed with the paired $t$ test or Wilcoxon signed rank test. A value of $P<0.05$ will be considered to be statistically significant. All analyses will be performed using SPSS (version 16.0. for Windows; SPSS Inc., Chicago, IL, USA).

\section{Ethical approval}

This study will be performed in accordance with the Declaration of Helsinki amended by the 59th World Medical Association General Assembly in 2008. All of the participants will sign a written informed consent stating that participation is voluntary and that participation can be withdrawn at any time, without any negative consequences concerning their current or future medical treatment. This study was approved by the Institutional Review Board of Seoul National University Bundang Hospital, Seoul National University Hospital, Seoul National University Boramae Medical Center, Hallym University Sacred Heart Hospital, and Kangnam Sacred Heart Hospital. The trial protocol has been registered at http:// www.clinicaltrials.gov (NCT01796418).

\section{Discussion}

Diabetic nephropathy is the number one cause of ESRD. CV complications are the most common cause of death in ESRD. Therefore, the early detection of CV risk using biomarkers and the prevention of CV risk with an effective drug are the only ways to improve the clinical outcomes and mortality in patients with diabetic nephropathy. BPS, a $\mathrm{PGI}_{2}$ analog, has shown many beneficial effects in both preclinical and clinical studies. In this trial, we will test whether BPS improves CV risk. This trial is the first to investigate the effects of BPS on changes in CV biomarkers, albuminuria, renal function, and lipid profiles in patients with diabetic nephropathy.

Because BPS has the potential to increase bleeding risk by inhibiting platelet aggregation, even patients on a single antiplatelet agent appear to be in danger of bleeding after their enrollment in this study. However, higher doses of BPS administered for a longer duration compared with this study were previously reported to not increase bleeding risk. According to the study performed by Mohler and colleagues [21], daily intake of $120 \mu \mathrm{g}$ BPS with other antiplatelet agents (aspirin, clopidogrel, or ticlopidine) for 1 year was not associated with any bleeding event. Furthermore, we will monitor and follow-up all adverse events, including bleeding, for the duration of the study or until resolution.

A 12-week study may be too short to demonstrate a beneficial effect of BPS. To assess the sustained effects of BPS on vascular benefits, long-term studies may be needed. However, in New York Heart Association functional class II and III patients with pulmonary arterial hypertension, a 12-week treatment with BPS improved exercise capacity and symptoms [22]. Moreover, the main mechanism of BPS in the improvement of arterial stiffness is through NO production, and BPS has a short half-life [12]. We therefore hypothesize that short-term treatment with BPS will improve arterial stiffness in this study. If the beneficial effects of BPS are proven, BPS could be used in clinical practice to improve CV and renal outcomes in this high-risk population. Future clinical trials with an extended administration of BPS over 52 weeks in a larger population will be required to determine whether BPS improves the actual CV morbidity and mortality.

\section{Trial status}

This trial is ongoing. Participants are recruiting.

\section{Abbreviations}

ABI: Ankle-brachial index; ba-PWV: Brachial-ankle pulse wave velocity; BPS: Beraprost sodium; CKD: Chronic kidney disease; Cr: Creatinine; CRF: Clinical research form; CV: Cardiovascular; eGFR: estimated glomerular filtration rate; ESRD: End-stage renal disease; ITT: Intention-to-treat; NO: Nitric oxide; $\mathrm{PGI}_{2}$ : Prostacyclin; PP: Per protocol; PWV: Pulse wave velocity; SAE: Serious adverse events. 


\section{Competing interests}

The authors declare that they have no competing interests.

\section{Authors' contributions}

KYN participated in the design of the study and drafted the manuscript. DKK conceived of the study, participated in the design of the statistical analysis, and helped to draft the manuscript. SGK and YKL participated in the design of the study. CSL conceived of the study, participated in acquiring funding, and had final responsibility for the decision to submit for publication. All authors read and approved submission of the final manuscript.

\section{Acknowledgements}

The authors would like to thank Songyi Han for her assistance with participant recruitment and data collection.

\section{Author details}

${ }^{1}$ Seoul National University Bundang Hospital, 82 Gumi-ro 172 Beon-gil, Seongnam-si, Gyeonggi-do 463-707, South Korea. ${ }^{2}$ Department Internal Medicine, Seoul National University College of Medicine, 103 Daehak-ro, Jongno-gu, Seoul 110-799, South Korea. ${ }^{3}$ Seoul National University Hospital, 101 Daehak-ro Jongno-gu, Seoul 110-744, South Korea. ${ }^{4}$ Hallym University Sacred Heart Hospital, 896 Pyeongchon-dong, Dongan-gu, Anyang, Gyeonggi-do 431-070, South Korea. ${ }^{5}$ Kangnam Sacred Heart Hospital, 948-1 Daerim1 dong, Yeongdeungpo-gu, Seoul 150-950, South Korea. ${ }^{6}$ Seoul National University Boramae Medical Center, 20 Boramae-ro 5-gil, Dongjak-gu, Seoul 156-707, South Korea.

Received: 14 June 2013 Accepted: 22 August 2013

Published: 2 September 2013

\section{References}

1. Collins AJ, Foley RN, Chavers B, Gilbertson D, Herzog C, Johansen K, Kasiske B, Kutner N, Liu J, St Peter W, Guo H, Gustafson S, Heubner B, Lamb K, Li S, Li S, Peng Y, Qiu Y, Roberts T, Skeans M, Snyder J, Solid C, Thompson B, Wang C, Weinhandl E, Zaun D, Arko C, Chen SC, Daniels F, Ebben J, et al: United States renal data system 2011 annual data report: atlas of chronic kidney disease and end-stage renal disease in the United States. Am J Kidney Dis 2012, 59(Suppl 1):e1-e420.

2. Jin DC, Ha IS, Kim NH, Lee SW, Lee JS, Yoon SR, Kim BS: Brief report: renal replacement therapy in Korea, 2010. Kidney Res Clin Pract 2012, 31:62-71.

3. Gaede $\mathrm{P}$, Lund-Andersen $\mathrm{H}$, Parving HH, Pedersen O: Effect of a multifactorial intervention on mortality in type 2 diabetes. N Engl J Med 2008, 358:580-591.

4. Gaede P, Vedel P, Larsen N, Jensen GV, Parving HH, Pedersen O: Multifactorial intervention and cardiovascular disease in patients with type 2 diabetes. N Engl J Med 2003, 348:383-393.

5. Gaede P, Vedel P, Parving HH, Pedersen O: Intensified multifactorial intervention in patients with type 2 diabetes mellitus and microalbuminuria: the Steno type 2 randomised study. Lancet 1999, 353:617-622.

6. Go AS, Chertow GM, Fan D, McCulloch CE, Hsu CY: Chronic kidney disease and the risks of death, cardiovascular events, and hospitalization. N Engl J Med 2004, 351:1296-1305.

7. Rubin MF, Rosas SE, Chirinos JA, Townsend RR: Surrogate markers of cardiovascular disease in CKD: what's under the hood? Am J Kidney Dis 2011, 57:488-497.

8. Blacher J, Guerin AP, Pannier B, Marchais SJ, London GM: Impact of aortic stiffness on survival in end-stage renal disease. Circulation 1999, 99:2434-2439.

9. Shoji T, Emoto M, Shinohara K, Kakiya R, Tsujimoto Y, Kishimoto H, Ishimura E, Tabata T, Nishizawa Y: Diabetes mellitus, aortic stiffness, and cardiovascular mortality in end-stage renal disease. J Am Soc Nephrol 2001, 12:2117-2124.

10. Mohler ER 3rd: Peripheral arterial disease: identification and implications. Arch Intern Med 2003, 163:2306-2314.

11. Melian EB, Goa KL: Beraprost: a review of its pharmacology and therapeutic efficacy in the treatment of peripheral arterial disease and pulmonary arterial hypertension. Drugs 2002, 62:107-133.

12. Sugawara A, Kudo M, Saito A, Matsuda K, Uruno A, Ito $S$ : Novel effects of beraprost sodium on vasculatures. Int Angiol 2010, 29:28-32

13. Goya K, Otsuki M, Xu X, Kasayama S: Effects of the prostaglandin I2 analogue, beraprost sodium, on vascular cell adhesion molecule-1 expression in human vascular endothelial cells and circulating vascular cell adhesion molecule-1 level in patients with type 2 diabetes mellitus. Metabolism 2003, 52:192-198.
14. Nakayama T, Hironaga $T$, Ishima $H$, Maruyama $T$, Masubuchi $Y$, Kokubun $S$ : The prostacyclin analogue beraprost sodium prevents development of arterial stiffness in elderly patients with cerebral infarction. Prostaglandins Leukot Essent Fatty Acids 2004, 70:491-494

15. Nakayama T, Masubuchi Y, Kawauchi K, Masaki R, Hironaga T, Ishima H, Torigoe M, Shimabukuro H: Beneficial effect of beraprost sodium plus telmisartan in the prevention of arterial stiffness development in elderly patients with hypertension and cerebral infarction. Prostaglandins Leukot Essent Fatty Acids 2007, 76:309-314.

16. Watanabe M, Nakashima H, Ito K, Miyake K, Saito T: Improvement of dyslipidemia in OLETF rats by the prostaglandin I(2) analog beraprost sodium. Prostaglandins Other Lipid Mediat 2010, 93:14-19.

17. Sato N, Kaneko M, Tamura M, Kurumatani H: The prostacyclin analog beraprost sodium ameliorates characteristics of metabolic syndrome in obese Zucker (fatty) rats. Diabetes 2010, 59:1092-1100.

18. Shin S, Kim KJ, Chang HJ, Lee BW, Yang WI, Cha BS, Choi D: The effect of oral prostaglandin analogue on painful diabetic neuropathy: a double-blind, randomized, controlled trial. Diabetes Obes Metab 2013, 15:185-188.

19. Levey AS, Coresh J, Greene T, Stevens LA, Zhang YL, Hendriksen S, Kusek JW, Van Lente F, Chronic Kidney Disease Epidemiology Collaboration: Using standardized serum creatinine values in the modification of diet in renal disease study equation for estimating glomerular filtration rate. Ann Intern Med 2006, 145:247-254.

20. Noordzij M, Tripepi G, Dekker FW, Zoccali C, Tanck MW, Jager KJ: Sample size calculations: basic principles and common pitfalls. Nephrol Dial Transplant 2010, 25:1388-1393.

21. Mohler ER 3rd, Hiatt WR, Olin JW, Wade M, Jeffs R, Hirsch AT: Treatment of intermittent claudication with beraprost sodium, an orally active prostaglandin 12 analogue: a double-blinded, randomized, controlled trial. J Am Coll Cardiol 2003, 41:1679-1686.

22. Galiè $N$, Humbert $M$, Vachiéry JL, Vizza $C D$, Kneussl $M$, Manes $A$, Sitbon $O$, Torbicki A, Delcroix M, Naeije R, Hoeper M, Chaouat A, Morand S, Besse B, Simonneau G, Arterial Pulmonary Hypertension and Beraprost European (ALPHABET) Study Group: Effects of beraprost sodium, an oral prostacyclin analogue, in patients with pulmonary arterial hypertension: a randomized, double-blind, placebo-controlled trial. J Am Coll Cardiol 2002, 39:1496-1502.

doi:10.1186/1745-6215-14-275

Cite this article as: Na et al.: Effect of beraprost sodium on arterial stiffness in patients with type 2 diabetic nephropathy. Trials 2013 14:275.

\section{Submit your next manuscript to BioMed Central and take full advantage of:}

- Convenient online submission

- Thorough peer review

- No space constraints or color figure charges

- Immediate publication on acceptance

- Inclusion in PubMed, CAS, Scopus and Google Scholar

- Research which is freely available for redistribution 